\title{
COMPARISON OF ULTRASOUND GUIDED MODIFIED PECTORAL NERVE BLOCK WITH LOCAL ANAESTHESTIC INFILTRATION FOR ANALGESIA IN BREAST CANCER SURGERY
}

\author{
Usman Saqib, Rashid Iqbal, Sana Usman, Muhammad Rashid Hanif \\ Combined Military Hospital/National University of Medical Sciences (NUMS) Rawalpindi Pakistan
}

\begin{abstract}
Objective: To compare the frequency of pain and mean analgesia requirement after breast surgery under general anaesthesia when comparing pectoral nerve block with local anaesthesia infiltration.

Study Design: Comparative prospective study.

Place and Duration of Study: Combined Military Hospital, Rawalpindi, from Mar 2018 to Sep 2019.

Methodology: A total of 60 patients undergoing modified radical mastectomy were included in the study. Group A ( $\mathrm{n}=30$ ) received pectoral nerve blocks while Group B $(n=30)$ received local anaesthetic infiltration. Outcome was assessed at 12 hours after injection of local anaesthetic.

Results: Pain in 7 (23.33\%) patients in Group A (Pectoral nerve blocks) and in 20 (66.67\%) patients in Group B (Local anaesthetic infiltration), $p$-value of 0.001 which was considered statistically significant. Mean analgesic requirement was found to be $80 \pm 33.73 \mathrm{mg}$ in the Group A (pectoral nerve blocks) compared with $141.67 \pm 47.50 \mathrm{mg}$ in Group B (Local anaesthesia infiltration) patients with a $p$-value of $<0.001$ which was statistically significant.

Conclusion: Pectoral nerve block significantly reduces early postoperative pain and analgesia requirement when compared with Local anaesthesia infiltration after breast surgery.
\end{abstract}

Keywords: Breast cancer surgery, Local anaesthetic, Pectoral nerve blocks, Post-operative pain.

How to Cite This Article: Saqib U, Iqbal R, Usman S, Hanif MR. Comparison of Ultrasound Guided Modified Pectoral Nerve Block with Local Anaesthestic Infiltration for Analgesia in Breast Cancer Surgery. Pak Armed Forces Med J 2021; 71(6): 1981-1984. Doi: https://doi.org/10.51253/pafmj.v6i6.4110

This is an Open Access article distributed under the terms of the Creative Commons Attribution License (https://creativecommons.org/licenses/by-nc/4.0/), which permits unrestricted use, distribution, and reproduction in any medium, provided the original work is properly cited.

\section{INTRODUCTION}

Analgesia is a significant domain of perioperative anaesthetic management and acute postoperative pain control results in poor surgical outcomes leading to prolonged hospital stays and patient dissatisfaction. ${ }^{1}$ Opioids and non-steroidal anti-inflammatory drugs, being components multimodal analgesic ladder, provide effective analgesia, however, they have significant adverse effects profile, especially having limitations in the pediatric and geriatric age group. Therefore, administration of local anaesthetics in epidural space is emergingon account of early mobilization, suppressing stress response and early post-operative recovery. ${ }^{2}$

Post-operative pain is the most common complication of any surgery. Soomer et al. stated $41 \%$ prevalence of moderate to severe pain after surgery despite of following acute pain protocols. Inadequately treated, acute postoperative pain may progress to chronic post-surgical pain (CPSP). ${ }^{3}$ Prevalence of chronic postsurgical pain is stated to be $14.8 \%$ in patients after general surgery. ${ }^{4}$

Breast cancer is the commonest carcinoma among

Correspondence: Dr Usman Saqib, Department of Anaesthesia, Combined Military Hospital Lahore Pakistan

Received: 13 Apr 2020; revision received: 07 Aug 2020; accepted: 17 Aug 2020 women with a prevalence of 1 in 9 women in Pakistan becoming a victim ofthis disease. ${ }^{5}$ Modified radical mastectomy (MRM) is a common surgical procedure performed for breast cancer cure.Lumpectomy with axillary lymph nodes dissection can be performed in certain cases. Extensive dissection is painful postoperatively and may result in acute neuropathic pain. Acute neuropathic pain after mastectomy left undertreated can develop into Post mastectomy pain syndrome (PMPS). The prevalence of PMPS has been reported to range from $20-68 \%$ in patients of MRM. 6,7

Various regional techniques have been used to manage post-operative pain after mastectomy including multimodal analgesia, Local anaesthesia infiltration, intercostal nerve blocks, paravertebral nerve blocks and thoracic epidural. ${ }^{8}$ Local Anaesthetic Infiltration and multimodal analgesia are routinely employed to treatpost-operative pain in our set up.

The pectoral nerves block considered less invasive and novel technique which involves pectoral, intercostobrachial, intercostal and long thoracic nerves block for provision of adequate pain relief in acute postoperative period..$^{9}$ Bashady et al, stated that postoperative Morphine requirement was significantly less in the Pectoral nerve block group $(2.9 \pm 1.714 \mathrm{mg})$ compared to the control group $(6.9 \pm 1.861 \mathrm{mg}) .12$ out of $60(20 \%)$ 
in pectoral nerve blocks group and 36 out of $60(60 \%)$ in the control group had post-operative pain, therefore statistically significant difference was observed $(p$ $<0.001) .{ }^{10}$ They are reportedly very safe with no complications in the acute postoperative period. In our study we compared post-operative analgesia following use of Pectoral nerve blocks and compared it with Local anaesthesia infiltration as recommended in qualitative systemic review of Pectoral nerve blocks.

\section{METHODOLOGY}

Comparative prospective study conducted at Combined Military Hospital, Rawalpindi, from March 2018 to September 2019. Permission from the hospital ethical committee $(04 \mathrm{~A} / 03 / 2018)$ was obtained. A written informed consent was taken from patients enrolled in the study after explaining the purpose of study along with the advantages and disadvantages of each technique used. Patients were selected from the General Surgery OPD and Pre Anaesthesia Clinic.

Inclusion Criteria: Female patients, American Society of Anesthesiologists (ASA) status I and II, with normal coagulation profile and age range between 18 to 65 years were included.

Exclusion Criteria: Patients with Basal Metabolic Index (BMI) greater than 35, metastatic carcinomas, recurrent carcinoma of the breast who have undergone breast, history of other major pectoral region or chest wall surgery, infection at site of injection, history of allergy to local anaesthetics and pregnancy were excluded.

Study population was divided into two equal groups. The sample size was calculated to be 30 in each group, with a significance level (a) $5 \%$, power of the test $(1-\beta)$ was $95 \%$, anticipated population proportion P1-20\% 10 and P2-60\%.10 Non-probability consecutive sampling technique was used.

Patients in Group A received pectoral nerve blocks whereas Group B received Local Anaesthetic Infiltration. General Anaesthesia was induced by using Propofol $(2-2.5 \mathrm{mg} / \mathrm{kg})$, Nalbuphine $(0.1 \mathrm{mg} / \mathrm{kg})$ as per standard premedication and induction protocols followed by maintenance with Isoflurane 1-2 MAC. Mastectomy was performed by Consultant Breast Surgeon on all patients. At the start of surgery, patients in group A were given pectoral nerve blocks under ultrasound guidance. Local Anaesthetic was injected at interfacial plane of pectoralis major and minor targets the lateral and median pectoral nerves. ${ }^{11}$ Local Anaesthetic was also administered at interfacial plane of pectoralis minor and serratus anterior to block pectoral, intercostobrachial, intercostals (III-VI) and long thoracic nerve. ${ }^{12} 10 \mathrm{ml}$ of $0.25 \%$ bupivacaine in superficial pectoral nerve block and $20 \mathrm{ml}$ of $0.25 \%$ bupivacaine in deep pectoral nerve block was administered. All patients in group-B were given $30 \mathrm{ml}$ of $0.25 \%$ Bupivacaine as local anaesthesia infiltration around the wound margins by the surgical team towards surgery end. The dose of Bupivacaine did not exceed $2 \mathrm{mg} / \mathrm{kg}$ body weight. Postoperative analgesia in the form of 50 mg IV boluses of tramadol was administered strictly on patient demand. Postoperatively pain was surveyed with a Visual Analogue Scale (VAS) at 12 hours after surgery. VAS is a psychometric response scale for the estimation of reaction of emotional attributes of agony that cannot be estimated directly. In this scale subjects react to their degree of torment by demonstrating a situation on a ceaseless line between two endpoints (Figure). ${ }^{13}$

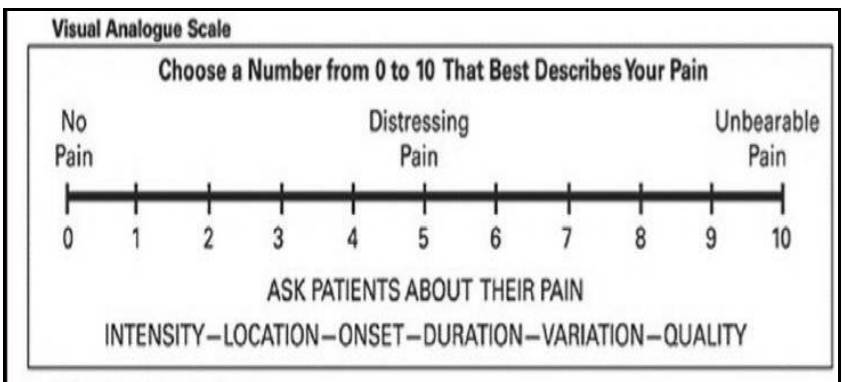

Figure: Visual analogue scale ${ }^{13}$.

Maximum dose of tramadol did not exceed 300 $\mathrm{mg}$ in 24 hours. Tramadol was recruited as postoperative analgesia on accountof ease of availability, confidence of inpatient facility staff in drug administration and better adverse effect profile. Outcome was assessed at 12 hours after injection of Local Anaesthetic.

Data was analyzed using Statistical Package for the social sciences (SPSS) version 23. For Qualitative, variables like no pain, frequency and percentages were used to measure frequency between two groups. For quantitative variables like analgesic consumption and age, mean and standard deviation (SD) were calculated. Independent sample t-test was used to compare analgesic consumption in both groups. Chi-square test was used to compare the frequency of pain between two groups. The $p$-value less or equal to 0.05 was considered significant.

\section{RESULTS}

Total sixty participants were enrolled in the study with equal distribution into two groups A (Pectoral nerve blocks) and B (Local anaesthesia infiltration). 
Patients were of age range from 18-65 years with a mean age being of $41.45 \pm 11.06$ years (Table-I). Out of these 60 patients, $26(43.33 \%)$ were ASA I and 34 (56.67\%) were ASA II. Mean BMI was $28.47 \pm 3.12 \mathrm{~kg} /$ $\mathrm{m}^{2}$. The mean BMI in group A was $28.27 \pm 3.19 \mathrm{~kg} / \mathrm{m}^{2}$ and in group B was $28.67 \pm 3.08 \mathrm{~kg} / \mathrm{m} 2$. Pain was found in $7(23.33 \%)$ patients in group A whereas 20 $(66.67 \%)$ group B $(p$-value $=0.001)$, statistically significant (Table-II). Mean analgesia requirement (Intravenous Tramadol) was found to be $80 \pm 33.73 \mathrm{mg}$ in the group A (Pectoral nerve blocks) compared with 141.67 $\pm 47.50 \mathrm{mg}$ in group B (Local anaesthesia infiltration) patients with $p$-value $<0.001$ (Table-III).

Table-I: Participants' age distribution $(n=60)$.

\begin{tabular}{l|c|c}
\hline \multirow{2}{*}{ Age (years) } & Group A (n=30) & Group B (n=30) \\
\cline { 2 - 3 } & Frequency (\%) & Frequency (\%) \\
\hline $18-40$ & $14(46.67 \%)$ & $12(40.0 \%)$ \\
\hline $41-65$ & $16(53.33 \%)$ & $18(60.0 \%)$ \\
\hline Mean \pm SD & $40.50 \pm 11.18$ & $42.40 \pm 11.05$ \\
\hline
\end{tabular}

Table II: Comparison of pain $(n=60)$.

\begin{tabular}{c|c|c|c|c}
\hline \multicolumn{2}{c|}{} & Group A (n=30) & Group B (n=30) & p-value \\
\hline \multirow{2}{*}{ Pain } & Yes & $07(23.33 \%)$ & $20(66.67 \%)$ & \multirow{2}{*}{$0.001^{*}$} \\
\cline { 2 - 4 } & No & $23(76.67 \%)$ & $10(33.33 \%)$ & \\
\hline
\end{tabular}

Table-III: Mean post-operative analgesia requirement.

\begin{tabular}{l|c|c}
\cline { 1 - 2 } Group A (n=30) & Group B (n=30) & \multirow{2}{*}{$\boldsymbol{p}$-value } \\
\cline { 1 - 2 } Analgesia Requirement & Analgesia Requirement & \\
\cline { 1 - 2 } Mean \pm SD & Mean \pm SD & \multirow{2}{*}{$<001^{*}$} \\
\hline $80 \pm 33.70$ & $141.67 \pm 47.49$ & \\
\hline
\end{tabular}

\section{DISCUSSION}

Pectoral nerve blocks and serratus plane blocks are newer regional anaesthesia modalities based on ultrasound guidance for pain relief in the surgeries of the thoracic region. Pectoral nerve block I targetsmedial and lateral pectoral nerves, innervating pectoralis muscles. In Pectoral nerve block II, a second injection is administered lateral to the Pectoral nerve block I anaesthetize intercostal nerves, administered in the plane between the pectoralis minor and serratus anterior muscles. Employed primarily for breast surgery, however, can be effectively used following thoracotomy and rib fracture. ${ }^{14}$

Wahba et al, in their study concluded that pectoral nerve block provides longer duration analgesia with reduced requirement of morphine, compared with thoracic paravertebral block. Used $30 \mathrm{ml}$ dosage of local anaesthetic in the pectoral nerve block whereas infiltrated $15-20 \mathrm{ml}$ thoracic paravertebral block at T4 level. However, due to relatively less dosage of local anaesthetic (Levobupivacaine 0.25\%) morphine was consumed considerably higher amount over 24 hours being
20-25 mg in Pectoral nerve block group and 22-31 mg in thoracic paravertebral block group. ${ }^{15}$

El-Sheikh et al, analyzed thoracic paravertebral block with Pectoral nerve blocks as pain management modality of breast surgery, and they concluded that there was the decreased requirement of intraoperative fentanyl in Pectoral nerve blocks group as compared with Thoracic vertebral block group. Moreover, the first dose of morphine was also reported to be delayed in Pectoral nerve block group. ${ }^{16}$

Yuki et al, used $0.25 \%$ levobupivacainepectoral nerve block versus general anaesthesia in carcinoma breast surgery. They concluded that fentanyl was consumed in significantly lower concentration over a period of 24 hours in the Pectoral nerve group than General anaesthesia group. ${ }^{17}$

Kulhari et al. determined the efficacy of pectoral nerve block versus thoracic paravertebral block as pain relief modality post breast surgery. They stated that participants were managed without fentanyl administration intraoperatively. As per their study, $60 \%$ nitrous oxide in oxygen and $0.5 \%$ ropivacaine in Pectoral nerve block and thoracic paravertebral block at T2-T3 levels provide improved analgesic efficacy without fentanyl consumption. ${ }^{18}$

Morioka et al. studied pain scores over a period of 48 hours postoperatively retrospectively, which were comparatively less in total intravenous anaesthesia augmented with Pectoral nerve block. ${ }^{19}$

It is recommended that pectoral nerve blocks should be used preferably in preventing postoperative pain after modified radical mastectomy to reduce the patient's morbidity.

\section{CONCLUSION}

Pectoral nerve blocks significantly reduce early postoperative pain and analgesic requirement as compared to local anaesthesia infiltration after breast cancer surgery.

\section{Conflict of Interest: None.}

\section{Authors' Contribution}

US: Conception, resarach analysis, data collection \& manuscript draft, RI: Supervisor, SA: Research analysis, data collection \& manuscript draft, MRH: Data collection.

\section{REFERENCES}

1. Fujiwara S, Komasawa N, Minami T. Pectoral nerve blocks and serratus intercostal plane block for intractable post-thoracotomy syndrome. J Clin Anesth 2015; 27(3): 275-276.

2. Bilgen S, Koner O, Menda F, Karacay S, Kaspar EC, Sozubir S. A comparison of two different doses of bupivacaine in caudal anaesthesia for neonatal circumcision. A randomized clinical trial. Middle East J Anesthesiol 2013; 22(1): 93-98. 


\section{Local Anaesthestic Infiltration}

3. Sommer M, Rijke J, Kleef M, Kessels AG, Peters ML. The prevalence of postoperative pain in a sample of 1490 surgical inpatients. Eur J Anaesth 2008; 25(4): 267-274.

4. Simanski CJ, Althaus A, Hoederath S, Kreutz KW, Hoederath P, Lefering $\mathrm{R}$, et al. Incidence of chronic postsurgical pain (CPSP) after general surgery. Pain Med 2014; 15(7): 1222-1229.

5. Menhas R, Umer S. Breast Cancer among Pakistani Women. Iran J Public Health 2015; 44(4): 586-587.

6. Jain PN, Padole D, Bakshi S. Prevalence of acute neuropathic pain after cancer surgery. Ind J Anaesth 2014; 58(1): 36-42.

7. Gartner R, Jensen MB, Nielsen J, Ewertz M, Kroman N, Kehlet H. Prevalence of and factors associated with persistent pain following breast cancer surgery. J Am Med Assoc 2009; 302(18): 19851992.

8. Madabushi R, Tewari S, Gautam SK, Agarwal A. Serratus anterior plane block: a new analgesic technique for post-thoracotomy pain. Pain Phys 2015; 18(3): E421-424.

9. Mejdahl MK, Andersen KG, Gärtner R, Kroman N, Kehlet H. Persistent pain and sensory disturbances after treatment for breast cancer: six year nationwide follow-up study. BMJ 2013; 346(1): f1865.

10. Bashandy GMN, Abbas DN. Pectoral Nerves I and II Blocks in multimodal analgesia for breast cancer surgery, a randomized clinical trial. Reg Anesth Pain Med 2015; 40(1): 68-74.

11. Blanco R. The "Pecs block": a novel technique for providing analgesia after breast surgery. Anaesth 2011; 66(9): 847-848.
12. Blanco R, Fajardo M, Maldonado PT. Ultrasound description of Pecs II (modified Pecs I): a novel approach to breast surgery. Rev Esp Anestesiol Reanim 2012; 59(9): 470-475.

13. Bourdel N, Alves J, Pickering G, Ramilo I, Roman H, Canis M. Systematic review of endometriosis pain assessment: how to choose a scale. Hum Reprod Update 2014; 21(1): 136-152.

14. Versyck B, Houwe PV, Geffen GJV, Velde VD, Slappendel R. A qualitative systematic review of the pectoral nerves block type I and II. Acta Anaesth Belg 2017; 68(2): 49-62.

15. Wahba SS, Kamal SM. Thoracic paravertebral block versus pectoral nerve block for analgesia after breast surgery. Egypt J Anaesth 2013; 30(2): 129-135.

16. Sheikh SM, Fouad A, Bashandy GN. Ultrasound-guided modified pectoral nerves block versus thoracic paravertebral block for perioperative analgesia in major breast surgery. Med J Cairo Univ 2016; 84(3): 189-195.

17. Yuki I, Ueshima H, Otake H. PECS Block Provides Effective Postoperative Pain Management for Breast Cancer Surgery. A Retrospective Study. Int J Clin Med 2017; 8(2): 198-203.

18. Kulhari S, Bharti N, Bala I. Efficacy of pectoral nerve block versus thoracic paravertebral block for postoperative analgesia after radical mastectomy: a randomized controlled trial. Br J Anaesth 2016; 117(3): 382-386.

19. Morioka H, Kamiya Y, Yoshida T, Baba H. Pectoral nerve block combined with general anaesthesia for breast cancer surgery: A retrospective comparison. JA Clin Rep 2015; 1(15): 15-18. 